A N N A L E S Annales de Bretagne et des Pays de l'Ouest

Anjou. Maine. Poitou-Charente. Touraine

123-4 | 2016

Varia

\title{
Landévennec, les Vikings et la Bretagne
}

\section{Philippe Guigon}

\section{OpenEdition}

Journals

Édition électronique

URL : http://journals.openedition.org/abpo/3446

DOI : $10.4000 / a b p o .3446$

ISBN : 978-2-7535-5365-1

ISSN : 2108-6443

Éditeur

Presses universitaires de Rennes

Édition imprimée

Date de publication : 30 décembre 2016

Pagination : 185-187

ISBN : 978-2-7535-5363-7

ISSN : 0399-0826

Référence électronique

Philippe Guigon, «Landévennec, les Vikings et la Bretagne ». Annales de Bretagne et des Pays de l'Ouest

[En ligne], 123-4 | 2016, mis en ligne le 30 décembre 2016, consulté le 23 septembre 2020. URL

http://journals.openedition.org/abpo/3446 ; DOI : https://doi.org/10.4000/abpo.3446 


\section{Comptes rendus}

Coumert, Magali, Tranvouez, Yvon (dir.), Landévennec, les Vikings et la Bretagne. En hommage à Jean-Christophe Cassard, Brest, Université de Bretagne Occidentale/ Centre de Recherche Bretonne et Celtique, 2015, 270 p.

"DCCCCXIII eodem anno destru[ctum est] monasterium sancti [Uuinwa]loei a Normannis ": cette simple annotation marginale d'un manuscrit conservé à la Bibliothèque royale de Copenhague, révélée par Léopold Delisle en 1890, offre l'occasion de rappeler la destruction de la plus ancienne abbaye bénédictine de Cornouaille, et de rendre hommage au regretté Jean-Christophe Cassard, décédé le 10 janvier 2013, exactement lors du $11^{\mathrm{e}}$ centenaire de ces ravages des Vikings. Cette doublement triste occurrence permet de rapprocher un site unique et ce regretté professeur de l'université de Bretagne occidentale, qui fut l'un des meilleurs spécialistes de ces aventuriers redoutables.

Le présent hommage ne doublonne pas celui qui a été publié sous la direction de Yves Coativy, Alain Gallicé et Laurent Héry, Jean-Christophe Cassard, historien de la Bretagne, Morlaix, Skol Vreizh, 2014. Après des siècles d'historiographie cléricale présentant ces peuples migrateurs comme des ennemis de Dieu et de la chrétienté, le besoin se faisait sentir de les observer de façon plus subtile, ce à quoi, pour la péninsule armoricaine, travailla notre collègue pour remettre largement en cause des idées énoncées dès le $\mathrm{IX}^{\mathrm{e}}$ siècle. Dans son introduction à l'ouvrage, Magali Coumert montre de quelle façon une bonne partie de l'œuvre scientifique de J.-C. Cassard s'articula autour des questions liées à la période des raids vikings, et ce dès 1986, avec la parution des Vikings en Bretagne, ouvrage réédité dix ans plus tard sous le titre Le siècle des Vikings en Bretagne. À la liste de ses travaux portant sur le haut Moyen Âge, il convient d'ajouter sa contribution "Les Vikings en Bretagne ", dans Les Vikings en France. Une synthèse inédite, Les Dossiers d'Archéologie, no 277, octobre 2002, p. 46-49. Sans minimiser le caractère sauvage des raids à répétition, J.-C. Cassard a montré de quelle façon ils "se trouvent sans doute à l'origine de la croissance économique continue qui animera l'Occident médiéval à partir du $\mathrm{XI}^{\mathrm{e}}$ siècle ", en mettant fin à la thésaurisation des objets précieux et en obligeant à la circulation monétaire qui facilita les échanges économiques.

Ceci étant, qu'en est-il plus particulièrement pour Landévennec, et quelle mémoire a été conservée de cette période? Répondre à cette question est l'objectif de cet ouvrage qui se décline en trois parties d'étendues inégales, un peu curieusement dénommées Structure, Conjoncture, Mémoire. La première s'ouvre par deux contributions d'archéologues et d'historiens de l'art, d'une part Annie Bardel et Ronan Pérennec, d'autre part Yves Gallet, à laquelle répond celle de l'historienne de la Cornouaille médiévale, Joëlle Quaghebeur. Tous s'attachent à décrire l'état du savoir sur l'abbaye, bien connue au Ix $\mathrm{x}^{\mathrm{e}}$ siècle, à la fois par des textes, mais également par des sources archéologiques qui permettent de remonter à des époques où l'histoire hésite à séparer mythe et réalité : pour résumer à grands traits, disons que Landévennec est l'abbaye européenne du IX ${ }^{\mathrm{e}}$ siècle qui 
semble correspondre le mieux à celle qui est dessinée sur le plan idéal de SaintGall vers 830. La Bretagne participe aux grands courants créatifs contemporains du continent, ce qui se vérifie pour d'autres réalisations, l'abbaye de Maxent et la cathédrale d'Alet. Notons qu'Yves Gallet date l'édifice bipolaire de la fin du $\mathrm{x}^{\mathrm{e}}$ siècle et celui qui lui est antérieur, à plan en tau, du siècle précédent, opinion que nous partageons mais qu'Anne Lunven a récemment contestée : pourtant, le plan de l'abbatiale de Landévennec de la seconde moitié du $\mathrm{IX}^{\mathrm{e}}$ siècle rappelle étonnamment celui de la cathédrale avec annexes situées de part et d'autre du chœur. L'un des apports important de la fouille de l'abbaye cornouaillaise est la mise au jour, à partir d'une sépulture privilégiée contiguë au chœur, de la pratique viking consistant à incinérer des ossements déplacés.

La deuxième partie de l'ouvrage, Conjoncture, met le cap au large sur la route des Vikings, de proche en proche : Noirmoutier (Cédric Jeanneau), les îles AngloNormandes (Élisabeth Ridel), Montreuil-sur-Mer (Stéphane Lebecq), enfin la Bretagne insulaire (Gary German). Les pérégrinations des reliques de saint Philibert, très bien connues (synthèse récente par Isabelle Cartron, 2010), évoluent de l'Herbauge (où l'abbatiale carolingienne de Saint-Philbert-de-Grand-Lieu a fait en 2015 l'objet d'un réexamen archéologique) jusqu'à la Bourgogne, trajectoire compliquée assez semblable à celles de saint Maixent. Plus risquée de prime abord, l'émigration des moines de Landévennec vers le Ponthieu où circulaient encore les Vikings. Stéphane Lebecq (directeur de l'édition en 2015 du cartulaire de Landévennec), après étude d'un corpus diplomatique peu épais mais complexe, conclut que, contrairement à l'hypothèse selon laquelle les moines de Fleury auraient amené à Montreuil-sur-Mer les reliques de Guénolé, les bénédictins cornouaillais se sont bien exilés en ce lieu, dans l'attente d'un passage jamais effectué vers l'Angleterre.

La dernière partie, Mémoire, pratique un grand écart chronologique entre les sources hagiographiques médiévales, et le $\mathrm{xx}^{\mathrm{e}}$ siècle. Dans la première contribution, André-Yves Bourgès examine ce que les Vies de saints et autres récits nous disent des Vikings, de façon rare, brève, et convenue. Les " Normands " y sont évidemment présentés sous le jour le plus noir, mais il est parfois possible de décrire la généalogie de ces textes édifiants, travestissant à leur manière les faits : ainsi, l'assassinat de l'évêque Gohard dans la cathédrale de Nantes, le 24 juin 843, est-il un topos hagiographique dont existent de multiples exemples, entre l'époque mérovingienne et... 1935 (Murder in the Cathedral, de Thomas Stearns Elliot). Un texte du cartulaire de Redon, dont Hubert Guillotel avait naguère démontré la falsification, évoque le destin du vir ferus, genere normannus Gurki, (un prototype de Gurguy/ Tanguy, vénéré $\mathrm{au} \mathrm{XV}^{\mathrm{e}}$ siècle par les Du Chastel en Léon?) : repenti, replié au fin fond de la presqu'île de Locoal qu'il dut restituer à Redon, cet " homme chien ", berserkr édenté, ne devait plus avoir beaucoup de mordant en 1027! Ces forgeries connurent une belle prospérité. Yvon Tranvouez, écrivant plaisamment que "le talent des médiévistes est de savoir faire quelque chose à partir de presque rien ", s'attache à retracer la résurrection par l'ineffable Arthur de La Borderie de l'abbé quasi-inconnu des textes Jean de Landévennec, mais surtout sa destinée théâtrale dans les années 1921 à 1937. S'il n'est pas certain que la pièce du chanoine François Cornou ait été propulsée en tête du box-office, la comparaison avec " une sorte de Jean Moulin unifiant la Bretagne libre et la résistance intérieure ", pour surprenante qu'elle soit, semble, une fois replacée dans le contexte de l'entre-deux-guerres, " une apologie intemporelle du fondement religieux de la nation " dont l'auteur décrit de façon passionnante les avatars.

À Landévennec au moins (la pièce fut jouée dans l'abbatiale le 4 septembre 1935, Xavier de Langlais tenant le rôle-titre), cette histoire laissa une trace, sous forme d'une plaque encore visible dans les années 1980, dénommant une placette 
du petit bourg, "Yann Landevenneg, libérateur de la Bretagne ". Mais, postérité éphémère, ce carrefour ne s'appelle plus aujourd'hui prosaïquement que "Place Yann Landevenneg ". Est-ce ainsi que les mythes meurent?

Philippe GUIGON

LEBECQ, Stéphane (dir.), Cartulaire de Saint-Guénolé de Landévennec, Rennes, PUR/ Société d'histoire et d'archéologie de Bretagne, coll. "Sources médiévales de l'histoire de Bretagne ", 2015, 456 p., avec des préfaces du père Jean-Michel Grimaud et de Ludovic Jolivet

La collection Sources médiévales de l'Histoire de Bretagne publiée par les PUR s'enrichit d'une nouvelle unité particulièrement prestigieuse : Le Cartulaire de SaintGuénolé de Landévennec. Ce fort volume de 450 pages ne se propose pas de rééditer le cartulaire, ce qui est chose déjà faite, mais, suivant la formule initiée par le Cartulaire de Redon puis celui de Quimperlé, d'offrir un fac-similé en photographies numériques accompagné d'un important dossier faisant le point sur les recherches autour du cartulaire et de l'abbaye. Inutile de souligner l'intérêt de cette publication concernant un monument de l'histoire de Bretagne. L'entreprise lancée dès 2006 par le CirDoMoc (Centre international de recherche et de documentation sur le monachisme celtique) n'a abouti qu'après bien des débats, des difficultés, et surtout après deux décès, celui du père Simon, infatigable historien de son abbaye auquel le volume est dédié, et celui de Bernard Merdrignac, l'une des chevilles ouvrières du CirDoMoc. Finalement, sous la direction de Stéphane Lebecq, professeur émérite d'histoire médiévale de l'Université de Lille 3 et grand spécialiste du haut Moyen Âge, ce volume a pu voir le jour avec le soutien de la Société d'histoire et d'archéologie de Bretagne, il s'agit donc bien d'une entreprise et d'une réussite collective s'il en fut.

Le choix de ne pas rééditer le cartulaire se comprend, l'ancienne édition de La Borderie, malgré ses imperfections demeure utilisable. Par contre, les diverses contributions qui accompagnent cette publication, souvent assez courtes, multiplient les angles d'approche du cartulaire, en éclairent le contexte sans oublier de restituer ce qu'était alors l'abbaye de Landévennec. Ainsi regroupés les différents textes apportent beaucoup à la compréhension du cartulaire en donnant accès au lecteur à des travaux qui se sont multipliés mais qui demeuraient le plus souvent inédits ou presque, comme toute l'archéologie de l'abbaye ou la thèse de Yves Morice sur les écrits de Landévennec.

Sébastien Barret présente d'abord, fort légitimement, une étude codicologique et paléographique du manuscrit conservé à la Bibliothèque de Quimper. Rédigé sous l'abbatiat d'Elisuc, au milieu du $\mathrm{xl}^{\mathrm{e}}$ siècle, le cartulaire ne va pas sans présenter une grande originalité. Il comprend, en effet, deux parties nettement inégales, une première qui occupe la grande majorité des pages se compose de textes hagiographiques et liturgiques à la gloire du fondateur, saint Guénolé, tandis que les chartes, souvent réduites à de courtes notices, voire à des extraits, n'occupent qu'une faible place. Cela témoigne de la conception du cartulaire selon ses auteurs : plus qu'une simple mise en forme des éléments du temporel, il s'agit bien d'une exaltation de l'abbaye et de son fondateur, assurant son identité et sa puissance. Si l'insertion de textes hagiographiques n'est pas unique (cf. le cartulaire de Sainte-Croix de Quimperlé), elle prend ici une place considérable et confère une originalité évidente au cartulaire, cette présence forte vient peut-être suppléer à un manque d'archives, les notices retenues 